\section{Praktisch ohne Alternative}

\section{Für die kommunale Verkehrsentwicklung kommł sowohl städtebaulich, planeri- schen Leitbildern als auch Verkehrsleitbildern eine tragende Rolle zu. Welche gibt es und gab es? Was sind ihre Merkmale? Woran wird Kritik geübt? Wo können Ansułzpunkte zur koordinierten Leifbildentwicklung liegen?}

$\mathrm{I}$ Von Ulrich Hatzfeld n Zeiten, in denen sich Stadtentwicklung vornehmlich als eine lockere Reihung von flachen ,Visionen" aus dem Katalog internationaler Projektentwicklungsroutine darstellt, sind Wertdiskussionen, kritische Reflexionen und Leitbilder, die ja immer auch zu einer gewissen Selbstbindung beitragen, nicht sehr populär. Die verbreitete Beliebigkeit des theoretischen Hintergrundes raumbezogener Planung fördert abstrakte Ziele wie „Behauptung im globalen Wettbewerb“ oder "Nachhaltigkeit“ oder - noch eleganter - „offene“ und „flexible" Leitbilder.

\section{Leitbilder im Widerspruch}

Die Kritik an Leitbildern für die Stadtentwicklung ist schnell zusammengefaßt. Bei sich in Individualismus beziehungsweise Gruppenegoismen auflösenden Interessenstrukturen wird eine Verständigung über ein übergreifendes, für alle Interessengruppen verbindliches räumliches Entwicklungsmodell immer unwahrscheinlicher. Stattdessen bilden sich voneinander unabhängige Teilleitbilder heraus, die sich überlagern und widersprechen können. Eine solche Komplexität der Interessen führt nicht selten zu sehr allgemeinen Kompromissen. Hinzu kommt, daß der ständige bzw. beschleunigte Wechsel maßgeblicher Leitbilder den Verdacht nahelegt, daß sie gegebene Trends nur noch nachvollziehen und ihre Steuerungsfunktion verlieren. Zuweilen verbindet sich die Kritik an Planungsleitbildern auch mit einer generellen Planungsfeindlichkeit (,Ideologielastigkeit“, „Unplanbarkeit gesellschaftlicher Prozesse“). Der vermutlich wichtigste Einwand gegen langfristige Orientierungen in der Stadtplanung besteht in dem Vorwurf, Leitbilder seien zu unflexibel, um in einer sich globalisierenden und immer kürzer getakteten Gesamtentwicklung der Städte noch Bedeutung zu haben. Die wesentlichen Ziele von Leitbildern, Komplexitätsreduktion, Kon- senserzeugung, Zukunftsorientierung, seien so die Kritik - unter den aktuellen gesellschaftlichen und ökonomischen Rahmenbedingungen kaum noch umzusetzen. chen problematisch sind, gibt es praktisch keine wirkliche Alternative. Im folgenden wird untersucht, wie sich diese Leitbilder auf einen der wichtigsten Bedingungsfaktoren der Stadtentwicklung, nämlich den Verkehrssektor, auswirken. Ausgangspunkt ist die wiederholt aufgestellte Behauptung, die Fachplanung „Verkehr“ habe im Rahmen der Stadtentwicklung einen eigenständigen Stellenwert entwickelt und sich von den übergeordneten Zielsetzungen gelöst. In welchem (historischem) Verhältnis stehen Planungsleitbilder zu den jeweils zugeordneten Zielvorstellungen des Verkehrs? Über welche Merkmale verfügen die jeweiligen Leitbilder? Gibt es eine theoretische praxisrelevante Hierarchie zwischen planerischen und verkehrlichen Leitbildern? Wo könnten Ansatzpunkte zur koordinierten Leitbildentwicklung liegen?

Selbst wenn man nur den relativ kurzen Nachkriegszeitraum betrachtet, gibt es keinen Mangel an programmatischer Neuorientierung und Paradigmenwechsel, sowohl in der Stadtplanung als auch im Verkehr. Zu den entsprechenden Entwicklungsphasen existiert bereits eine Vielzahl an Analysen (1). Daher man sich heute auf die Leitbildbereiche Städtebau und Verkehr zu beschränken.

\section{- 50er Jahre - autogerechte Stadt}

Nahezu alle größeren deutschen Städte standen am Kriegsende vor den Entwicklungsoptionen „Restauration“ oder „Neugestaltung“" des überkommenen Stadtgrundrisses. Fast immer entschied man sich für die erste Entwicklungsalternative, wofür neben der Stabilität der Grundbesitzverhältnisse (fehlende Bodenreform) vor allem die Option zur Nutzung der noch weitgehend intakten Ver- und Entsorgungs-
Obwohl Leitbilder zwar theoretisch ausgespro- infrastrukturen in den Straßen maßgeblich war. Die vorherrschende Planungsidee der Wiederaufbauphase war die der, gegliederten und aufgelockerten Stadt"(2). Das an der Vision einer organischen Stadtlandschaft orientierte Leitbild greift wesentliche Elemente von Planungsideologien der 20er und 30er Jahre, in gewisser Weise auch des Nationalsozialismus, auf. Es legitimierte sich allerdings im Gegensatz zu diesen vorangehenden Leitbildern in erster Linie an „biologischen" Ansprüchen von Menschen. Die gegliederte und aufgelockerte Stadt war unter dem Gesichtspunkt der Verdichtung wenig urban, wandte sich gegen hohe Bebauungs- und damit Einwohnerdichten und propagierte das Prinzip der Funktionstrennung; vor allem ging es aber auch um eine gute Ausstattung mit Freiflächen.

Mit dem städtebaulichen Leitbild der 50er Jahre korrespondierte das verkehrliche Erschliessungssystem des reinen Verästelungsprinzips. Übergeordnet war die Vorstellung der ,autogerechten Stadt", die auch häufig die Grundlage der ersten, in dieser Zeit entstehenden Generalverkehrspläne bildete. Allerdings ging es dabei weniger um eine Unterordnung stadtplanerischer Belange unter Ansprüche des Verkehrsmittels Auto als vielmehr um eine Klärung der Entwicklungsoptionen, die sich durch die (verkehrs-) technischen Innovationen im Hinblick auf die angestrebten ,gesunden Lebensverhältnisse" ergaben. Stichworte waren „Trennung der Verkehrsarten", „Dezentralisierung" und "Auflösung der Stadt in die Stadtlandschaft". Folgerichtig mußte die für das Leitbild der aufgelockerten und gegliederten Stadt typische Siedlungsweise zu einer Präferierung des motorisierten Individualverkehrs (MIV) führen, schon allein deshalb, weil „lockere“ und gering verdichtete Siedlungsstrukturen durch Massenverkehrsmittel kaum wirtschaftlich $\mathrm{zu}$ erschließen sind.

In diese Zeit fallen auch die Anfänge der ,rasanten Verwissenschaftlichung der Verkehrsplanung", die sich durch einen starken Drang zur ingenieurgerechten Quantifizierung und prognostischen Besprechung ausdrückt und die für diese Fachplanung später prägend werden sollte.

\section{0 er Jahre - Verkehrsknotenpunkt Stadt}

In den 60er Jahren vollzog sich ein Wechsel vom Ziel der "Gliederung und Auflockerung" hin zur „Verdichtung und Verflechtung". Hintergrund für 
diesen Leitbildwechsel war eine heftige Kritik an den in den 50er Jahren entstandenen monofunktionalen und monotonen ,Schlafstädten“(3). Ungeachtet der Ziele der Verflechtung bzw. Mischnutzung setzte sich der bereits in den 50er Jahren begonnene Suburbanisierungsprozeß fort. Die räumliche Expansion von Gewerbe und Industrie sowie auch des verdichteten, um vielfältige Nutzungen angereicherten Wohnens vollzog sich primär als Stadterweiterung. Die Innenstädte wurden mit dem Schwerpunkt Verwaltung und kommerzielle Nutzung entwickelt. In die 60er Jahre fällt aber auch der Anfang der Stadterneuerung, ausgehend von Funktionsschwächen- und Flächensanierungen zur Beseitigung ökonomischer, funktionaler und stadtstruktureller Mißstände. In verkehrlicher Hinsicht wurden in dieser Entwicklungsperiode in den meisten großen Städten Generalverkehrspläne mit Stadtautobahnen und Programm zum Öffentlichen Personennahverkehr (ÖPNV) realisiert. Diese Pläne und Programme sind als erste Reaktion auf die sich in der Politik verstärkende Erkenntnis zu werten, daß eine systematische konzeptbezogene Entwicklung sowohl des Pkw-Verkehrs als auch der Massenverkehrsmittel erforderlich ist, um die sich dynamisch vergrößernden Verkehrsprobleme anzugehen. Zentraler Gegenstand der Stadtverkehrsplanung waren dabei die Innenstädte, die entsprechend dem ,ökonomisch-funktionalen Leitbild“ mit zum Teil großen Aufwand zu Verkehrsknotenpunkten ausgebaut wurden.

Da es für diese Entwicklungsperiode der Verkehrsplanung keinen einschlägigen Leitbildbegriff gibt, kann man diese Phase vielleicht mit den Begriffen „Optimierung und Kapazitätsausweitung" kennzeichnen. Es bildet sich eine Fachdisziplin heraus, die sich vor allem aus ingenieurwissenschaftliche Perspektive mit Phänomenen wie „Verkehrsaufkommen“, ,modalsplit" und „Verkehrsverteilung" befaßte, „Knotenpunkte und Straßenquerschnitte berechnete, Trendprognosen aufstellte, Generalverkehrspläne ausarbeitete, kurz, in ihren Planungen ihre eigenen fachspezifischen Irrtïmer beging“ (4).

\section{0 er Jahre bis heute - Vielfalt der Leitbilder}

Am Anfang einer nochmaligen städtebaulichen Leitbildveränderung Anfang bis Mitte der 70er Jahre stand wiederum eine grundlegende Kritik an der vorangegangenen Entwicklungsphase, die unter anderem unmaßstäbliche Massierungen und Maßstabsverzerrungen im Städtebau hervor- gebracht hatte. Stadterweiterung sollte - so die sich verstärkende Meinung - durch behutsamen Stadtumbau ersetzt werden. Im Gegensatz zu den 60er Jahren, in dem visionäre Leitbilder diskutiert wurden, setzen die 70er Jahre auf Konsolidierung und Regeneration, in gewisser Weise sogar auf Veränderungsverweigerung. Es geht nicht mehr um perfekte, auf lange Entwicklungsperioden hin orientierte Planungskonzepte und methoden, sondern um Einmaligkeit, Ortsbezogenheit und die Erhaltung künftiger Entwicklungsmöglichkeiten. Neben dem Postulat der ,behutsamen Erhaltung“ gewinnen auch die beiden Bewertungsdimensionen „Ökologie" und „geschichtliche Einordnung" an Bedeutung.

In der Folge ist ein Verlust von umfassenden, „übergeordneten“ Leitbildern zu konstatieren: Integrierte Leitbilder der Stadtentwicklung, die sich in Ziel- und Maßnahmenkataloge der Stadtentwicklungsplanung operationalisieren lassen, gibt es seitdem nicht mehr. Stattdessen entwickeln sich immer mehr partielle Leitbilder heraus, die sich auf einzelnen Sektoren oder städtische Teilbereiche (vor allem Innenstädte) beziehen (siehe Kasten). Gleichzeitig setzt eine neue Diskussion über die Definition städtischer Urbanität ein, über die ästhetische Dimension hinaus verbunden mit Ansätzen der Vielfalt, Vitalität und Nutzungsänderung.

Entgegen der einsetzenden Differenzierung der Städtebau- und Stadtentwicklungsleitbilder ist in Fragen der Leitvorstellungen zum Verkehrsbereich keine ähnliche Vielfalt entstanden. Vielmehr hat es den Anschein, als hätten sich in der Verkehrsplanung zwei Strömungen stabilisiert.

- Auf der einen Seite gibt es den Fortbestand einer eher an traditionellen Zielen wie „Leichtigkeit und Flüssigkeit" orientierten Auffassung von Verkehrsplanung, die sich vor allem auch mit den Möglichkeiten und Restriktionen der Verkehrstechnik befaßt. Die hier entwickelten Lösungen sind vornehmlich technischer organisatorischer Natur.

- Daneben entstand in Korrespondenz zur behutsamen Stadterneuerung das Konzept der Verkehrsberuhigung - einer der ersten expliziten Versuche, die Verkehrsfunktionen von Straßen in ein ausgewogenes Verhältnis zu anderen, „städtischen“ Funktionen von Verkehrswegen zu bringen. Aus der Verkehrsberuhigung entwickelte sich schirittweise die Konzeption der „stadtverträglichen Verkehrsplanung“ mit den drei Hauptstrategien „Verkehrsvermeidung, verlagerung und -beruhigung“.

\section{Der Einfluß des Verkehrs auf die Stadtentwicklung}

Wie aber sehen jenseits der Leitbilder die realen Bilder - unsere Städte und Verkehrswege - heute aus? Bereits vor mehr als 60 Jahren bemerkte der Hamburger Stadtbaurat Fritz Schumacher: „,... beim heutigen Zustand der Umentwicklung alter Städte münden alle ihre Fragen schließlich in Fragen der Verkehrspolitik ein, da sie die stärkste aller umgestaltenden Kräfte ist" (5). Tatsächlich hat kaum eine andere Rahmenbedingung der Stadt- und Regionalentwicklung das äußere Erscheinungsbild, das funktionale Gefüge und die Lebensbedingungen in Städten derart stark beeinflußt wie verkehrliche Faktoren. Jede größere Verkehrsinnovation hat letztlich zu einem neuen Stadttyp gefuihrt. Die Zusammenhänge sind bis in die Wurzeln der Stadtgeschichte nachgewiesen, waren besonders wirksam im Zeitalter der schienengebundenen Massenverkehrsmittel und enden vorläufig bei den kaum noch beherrschbaren Umweltimplikationen des privaten Personenverkehrs.

Vor diesem Hintergrund verwundert es zunächst, daß es für die beiden Handlungsbereiche Stadtentwicklung und Verkehr iberhaupt eigenständige, unterschiedliche Entwicklungsleitbilder gegeben hat oder noch gibt. Ferner erscheint bemerkenswert, daß zwischen den Leitbildern der Stadtentwicklung und des Verkehrs keine eindeutige Hierarchie in dem Sinne existiert, daß sich die verkehrlichen Leitbilder in die ,"̈̈bergeordneten“ Leitbilder der Stadtentwicklung einfügen bzw. daraus abgeleitet wurden. Dies hat sich allerdings in den letzten Jahren deutlich geändert: gerade in den 80er Jahren hat es eine Annäherung auf der Zielebene gegeben. Begriffe wie ,stadtverträgliche Verkehrsplanung" dokumentieren einen verstärkten Bezug der Fachplanung zur städtischen Gesamtplanung. Ähnliches gilt für Stadtentwicklungskonzeptionen, die unter den Leitmotiven „Nutzungsmischung“ und „Stadt der kurzen Wege" stehen und explizit aus dem Wunsch abgeleitet wurden, Mobilitätsanspriiche in der Stadt zu bewältigen.

Ungeachtet der Konvergenz von Verkehrs- und Stadtentwicklungsleitvorstellungen- kann nicht verschwiegen werden, daß der Bereich der Verkehrstechnik seinen Einfluß auf die Praxis der Verkehrsplanung nicht verloren hat. Auch heute noch sind Verkehrsplanungsstrategien, die auf eine bewußte Reglementierung von Verkehr zielen, nur gegen entschiedenen ,fachlichen Widerstand" durchzusetzen. So gibt es in der Verkehrsplanung neben dem zur Zeit aktuellen Mythos der Verkehrsvermeidung und -verlage- 
rung noch immer den informellen Mythos der Flüssigkeit und Leichtigkeit des Verkehrs. Uneinheitlich ist auch die öffentliche Auseinandersetzung über die Zukunft des Verkehrs: die zuweilen politisch geforderte "Verkehrswende“ ist offensichtlich noch weit von der Realität entfernt.

\section{Döffnung und Dialog erwünscht}

Sicher ist es im Interesse des Umsetzungsbezugs von Planung auch in Zukunft wichtig, den Unverbindlichkeitsgrad von Leitbildern zu verringern. Beiden Handlungsfeldern täte es gut, Prinzipiendiskussionen zu vermeiden und über gemeinsame Interessen nachzudenken:

- Häufig hat die Stadtplanung die Frage der Mobilität in Städten auf die Diskussion von Grundsätzen reduziert. Es geht aber weniger um „schöne“ und theoretisch einwand- freie Pläne als vielmehr darum, Bürger und Interessensvertreter von den (individuellen) Vorteilen einer autoverkehrsärmeren Stadt zu überzeugen. Anstelle einer abstrakten Diskussion über Verkehrsvermeidung sollten lieber konkrete Optionen geprift werden, wie ein veränderter Umgang mit Verkehrsmitteln zur Erhöhung der individuellen Lebensqualität führen kann. Dies erfordert Versuche und Experimente, denn neues Verkehrsverhalten wird vorrangig ein Resultat persönlicher Bewertung und individueller Erfahrung sein.

- Die traditionsreiche Verkehrswissenschaft sah sich in den letzten Jahren sehr häufig sozialwissenschaftlichen „Belehrungen“ ausgesetzt; dies hat nicht selten einen weiteren Rückzug auf den fachspezifischen Kern der Verkehrsplanung bewirkt. Dennoch bleibt ein Insistieren auf technische Zwänge

\section{Leitbilder zur Stadtentwicklung}

\section{- Revitalisierung der Innenstadt}

Dabei geht es in erster Linie um die Steigerung der Standortattraktivität, die - neveren Ansätzen folgend vor allem über eine Inszenierung von Innenstädten in gestalterischer Hinsicht erreicht werden soll. Im Zuge einer weitgehenden „Ästhefisierung" von stâdtebaulichen Problemen werden zentrale Entwicklungsfragen in Stilfragen uminterpreliert.

\section{- Collage City}

In Anlehnung der Arbeiten von C. Rowe verzichter die Planung ouf ihren übergeordneten Steuerungsanspruch und konzentriert sich ouf die Reclisierung von Fragmenten (Einzelprojekten). Eine solche weitgehende Projektorientierung prägt beispielsweise die Internationale Bauausstellung Emscher Pork.

\section{- Partizipation}

Die Interessensgebundenheit und politische Einseitigkeit von Planungsprozessen soll durch eine möglichst kompetente und umfassende Mitwirkung der Bürger (organisierte Öffentlichkeit) kontrolliert werden.

\section{- Kompakte Stadt und dezentrale Konzentration}

Durch eine Konzentration der Entwicklungsdynamik ouf bestimmte Punkte (Entwicklungsschwerpunkte) und Linien (Achsen) soll Zielen wie Verkehrsvermeidung und Freiroumschutz zur Umsetzung verholfen werden. Zentrale Instrumente sind die Erzeugung von Nutzungsmischung, Dichte und Vernetzung (,kompakte Stadt").

\section{- Nachhaltige Stadt}

Spätestens seit der Rio-Konferenz zu Umwelt und Entwicklung Anfang der 90 er Jahre beziehen sich viele Pla- nungsstrategien auf das sogenannte sustainable development. Diese zukunftsorientierte Entwicklungsstrotegie zielı ouf die Umsetzung von Prinzipien wie Reversibilität (Möglichkeit der Umkehrung von Prozessen), Regeneration (Wiederherstellbarkeit eines Ursprungszustandes), Substituierbarkeit (Möglichkeit zum Ersalz von Stoffen usw.), räumliche Betroffenheit (Berücksichtigung des räumlichen Bezugs), zeitliche Betroffenheit (Begrenzung der Daver von Belastungen), gesellschaftliche Betroffenheit (Bürgerbeteiligung) sowie psychische und physische Betroffenheit (6). Die städtebauliche Konkretisierung de Nachhaltigkeit stellt in erster Linie die Konzeption der Nutzungsmischung dar: Innenentwicklung und Nutzungsmischung gelten vor allem auch deshalb als nachhaltig, weil Beiträge für verkehrssparsame Siedlungsweisen erwartet verden.

\section{- Stadt der globalen Investition}

Moderne Entwicklungen wie international orientierte Ansiedlungsstrategien, weltweite Informationsnetze und die globale Akquisition von Arbeit und Kapital erfordern nach Ansicht vieler eine neue Ebene der Kooperation von Städten und Regionen mit dem internationalen Kapital. Ziele sind die Erhöhung der regionalen Wettbewerbstähigkeit bzw. die Steigerung des wirtschaftlichen Wachstums. Die Rolle der Stadplanung reduziert sich dabei auf eine möglichst rasche Bereitstellung der erforderlichen Infrastruktur und der "Abfederung" möglicher sozioler Konflik te, die durch die wirtschaftliche Entwicklung induziert werden. Zentroler Aspekt eines solchen Leitbildes ist dabei die flexiblere Ermöglichung von Wandel, also auch ein Verzicht auf räumlich verfestigte Leitvorstel. lungen. und Normen, die sich die Verkehrsplanung letztlich selber geschaffen hat, langfristig ohne Perspektive. Eine öffnung der Verkehrsplanung für eine „Wertediskussion“ ist wichtiger denn je, weil sich auch eine Fachwissenschaft heute nicht mehr Grundsatzfragen wie der Erhaltung von Natur und Landschaft oder der ökologischen Lebensgrundlagen entziehen kann. Die in der Vergangenheit sicher erfolgreiche Strategie, Verkehrswachstum mit Infrastrukturwachstum und technischer Verbesserung $\mathrm{zu}$ begegnen, wird in absehbarer Zukunft schon allein aus Finanzierangsgründen nicht mehr tragen.

Denkt man über die gemeinsame Zukunft von Stadtentwicklung und Verkehr nach, wird man sich schnell mit Grundsatzfragen befassen müssen. Dazu gehört der Umgang mit Raum (z.B. „Neubewertung der Nähe und des Örtlichen“) und der Umgang mit Zeit (,Neubewertung von Geschwindigkeit", ,Verkürzung von Innovationszyklen"), immer bezogen auf die Qualität des Lebens in Städten. Wir stehen erst am Anfang dieser notwendigen Diskussion.

\section{Anmerkungen}

1) vgl. v.a.: H.R. Müller-Ramisch (Hrsg.): Leitbilder und Mythen in der Stadtplanung 1945-1985. Frankfurt /M. 1990

- Wüstenrot Stiffung Deutscher Eigenheimverein e.V.: Zukunft Stadt 2000: Stand und Perspektiven der Stadtentwicklung.

- D. Reinborn: Städtebau im 19. Und 20. Jahrhundert. Stuttgart, Berlin, Köln 1996

2) J. Göderitz, R. Rainer, H. Hoffmann: Die gegliederte und aufgelockerte Stadt. Tübingen 1957

3) vgl. die Arbeiten von Bahrdt zum Thema "Öffentlichkeit und öffentlicher Raum", Jane Jacobs oder Alexander Mitscherlich

4) W. Pehnt: Die Erfindung der Geschichte. München 1989 5) U. Riedel (Hrsg.): Erlebnisraum Innenstadt - Beiträge zU einem Verkehrs-, Wirtschafts- und Kulturkonzept für Bremen. Bremen 1990, S. 8-11

6) G. Arlt, S. Siedentop: Grundzüge eines sustainability-Konzepts für die Siedlungs- und Flächenentwicklung in Großstadtregionen. in: K. Brake, U. Richter (Hrsg.): Sustainable development, Ausgangsüberlegungen zur Theorie einer nachhaltigen Stadtentwicklung. Oldenburg 1996, S. 13-30

Der Autor
Dr. Ulrich Hatzfeld, Stadt-, Regional- und Landes-
planer im Ministerium für Stadientwicklung, Kultur
und Sport des Landes Nordrhein-Westfalen,
40190 Düsseldorf, Tel. $(02111) 837-4468$


(c) 20I0 Authors; licensee IÖW and oekom verlag. This is an article distributed under the terms of the Creative Commons Attribution Non-Commercial No Derivates License (http://creativecommons.org/licenses/by-nc-nd/3.o/), which permits unrestricted use, distribution, and reproduction in any medium, provided the original work is properly cited. 